\title{
Normal Contact Damping Predict Model of Joint Surface Based On Unit Area and Fractal Theory with Simulation
}

\author{
Yongsheng Zhao ${ }^{a}$, Yi Hong ${ }^{\text {b }}$ \\ Beijing University of Technology, Beijing 100124, China \\ azys.swm@gmail.com, byiscar@126.com
}

\begin{abstract}
Keywords: Joint surface, Fractal contact theory, Normal contact damping, Area ratio, Predict Model, Numerical simulation.
\end{abstract}

\begin{abstract}
Joint surfaces widely exist in mechanical assembly structures, the local stiffness and damping have important effect on the whole dynamic analysis of the structure. In this study, proposed a normal contact damping forecast model and analyze its damping characteristics, based on the microcosmic mechanism between joint surfaces and citing the domain extension factor and unit area radio, combine with the modified fractal theory and energy dissipation. The simulation results show that the damping characteristic curve appear inflection point when the fractal dimension value $\mathrm{D}$ is 1.428. When, the normal contact damping increases with the increase of fractal dimension but decreases with the increase of contact area. When, the normal contact damping increase with the increase of contact area. Since the reaction of real contact area is relate to the change of the load, the relationship between the normal contact damping and the contact area can accurately predict the performance of assembly structure to a certain extent, the existence of the inflection point also provide a reference for the optimization design of assembly structure.
\end{abstract}

\section{Introduction}

Joint surfaces is the assembly structure main source of nonlinear damping, it has very important influence on the dynamic behavior of the whole structure, joint surface cannot be ignored in the study of the mechanical structure dynamic characteristics.[1]. Under normal circumstances, the joint surface contact damping accounted for more than $90 \%$ of the total structure damping [2], compared with mechanical parts itself, the joints contact damping the dominant element. It has important significance in the whole assembly structure dynamics analysis and structure optimization design by researching joint surface contact damping. Due to the contact problem between the rough surfaces is nonlinear and complicated, the joint surface contact damping parameters usually got by the method of experiment to identify, in the former studies. [3-4], with the development of fractal contact theory application in rough surface [5], researchers began to expand the research field of fractal contact model in the field of bolt joint dynamic characteristic. References [6-8] researched the joint surface contact stiffness contact damping based on two-dimensional fractal surface, and the corresponding fractal model is established. In this paper, a normal contact damping forecast model is established, the influence of surface parameters on the normal contact damping is also analyzed by digital simulation. The simulation inflection point value is bigger than the reference result which is 1.42 [9].

\section{Fractal contact theory and micro-contact model}

\subsection{Fractal contact theory}

The contact of two rough surfaces can be equivalent as a rough surface contact with a rigid plane. On the micro scale, the rough surface is composed of a large number of asperities, and the shape of the rough peak is usually oval. Since the oval contact region size is far less than the radius of curvature of itself, the single rough peak can be approximated as sphere.

When two joint surfaces contact and squeeze each other, the higher asperity on rough surface will be the first to occur deformation, as shown in figure 1.A single asperity contact with the rigid surface is shown in figure 2 : 


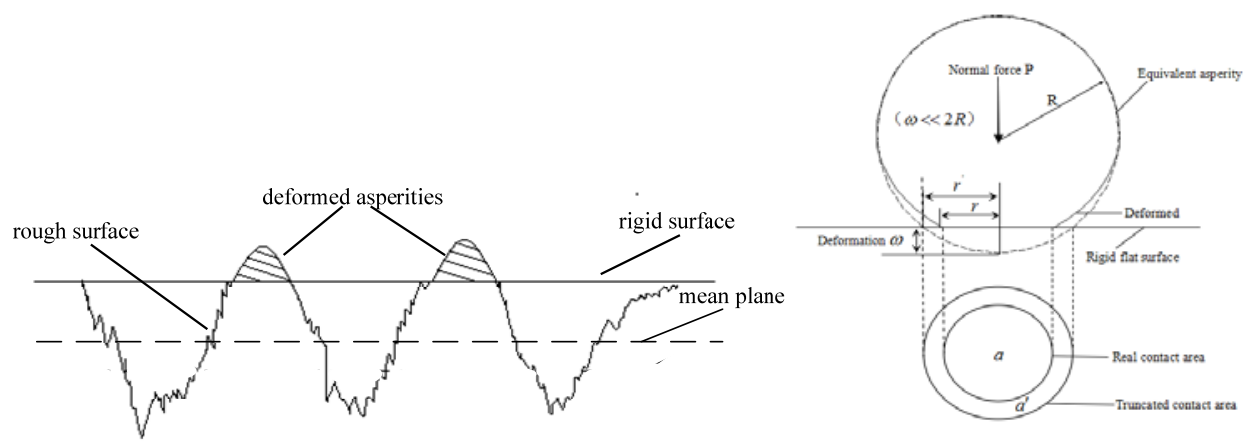

Fig.1 Rough surface contact with the rigid flat Fig.2 Asperity elastic contact with the rigid schematic flat

The rough surface profile is random and disordered, performance continuous, no differentiable and statistically self-affine properties in mathematics. The Weierstrass-Mandelbrot function which satisfies all of the above properties can be given as [10]:

$$
z(x)=G^{(D-1)} \sum_{n=n_{1}}^{n_{\max }}\left(\frac{\cos 2 \pi \gamma^{n} x}{\gamma^{(2-D) n}}\right) ; 1<D<2 ; \gamma>1 ; \gamma^{n 1}=1 / L
$$

Where the parameter $D$ is the fractal dimension, $G$ is the fractal roughness parameter, $\gamma^{n}$ determines the frequency spectrum of the surface roughness, $L$ the length of the sample.

Wang and Komvopoulos [11] introduced the domain extension factor $\psi$ for micro-contact size distribution function, and obtained the ratio of the maximum real contact area $a_{l}$ for one single asperity of joint interface to total real contact area $A_{r}$. The more accurate size distribution function of micro-contact was given as:

$$
n\left(a^{\prime}\right)=\frac{D}{2} \psi^{(2-D) / 2} a_{l}^{\prime D / 2} a^{\prime-(2+D) / 2} \quad 0<a^{\prime} \leq a_{l}^{\prime}
$$

Where $a^{\prime}$ is the truncated area of micro-contact, $a_{l}^{\prime}$ is the largest truncated area of the micro-contact, $\psi$ is the domain extension factor, which is a function of fractal dimension and can be given as

$$
\frac{\psi^{(2-D) / 2}-\left(1+\psi^{-D / 2}\right)^{-(2-D) / D}}{(2-D) / D}=1 \quad \psi>1
$$

The asperity interference $\omega$ for the micro-contact with the truncated area $a^{\prime}$ can be expressed as $\omega=G^{(D-1)} \cdot a^{\prime(2-D) / 2}$

The equivalent asperity curvature radius of the micro-contact $R$ can be express as

$$
R=\frac{a^{\prime D / 2}}{\pi^{2} G^{D-1}}
$$

\subsection{Micro-contact model}

The classic Hertz elastic contact theory is based on the assumption of contact area small deformation and no friction, and each asperity is treated as elastic half space and do not consider the interaction between each other. [12] The elastic deformation of a single asperity under normal force is

$$
P=\frac{4}{3} E^{*} R^{1 / 2} \omega^{3 / 2}
$$

Where $E^{*}$ represents the equivalent elastic modulus of the contact materials, satisfied $E^{*}=\left[\left(1-v_{1}^{2}\right) / E_{1}+\left(1-v_{2}^{2}\right) / E_{2}\right]^{-1}$, and $E_{1}, v_{1}$ and $E_{2}, v_{2}$ are the elastic modulus and Poisson's ratio of rough surface and rigid surface, respectively.

The pressure of the single asperity increase with the increase of load between two surfaces, and even lead the asperity to be yield. The critical interference $\omega_{c}$, critical contact radius $r_{c}$ and the critical load of inception of plastic deformation $P_{c}$, was defined by Chang et al. [13] by using the von Mises yield criterion. The resulting equation is 


$$
\begin{aligned}
& \omega_{c}=\left(\frac{\pi K H}{2 E^{*}}\right)^{2} R \\
& r_{c}=\frac{\pi K H R}{2 E^{*}} \\
& P_{c}=\frac{2}{3} K H \pi \omega_{c} R
\end{aligned}
$$

Where $H$ represents the hardness of softer material, $K$ is the coefficient of hardness, which satisfied $K=0.454+0.41 v$, and $v$ is the Poisson's ratio of softer material.

Kogut and Etsion (2004) quantitatively distinguish the contact deformation through the dimensionless deformation $\omega^{*}$. Where, $\omega^{*}$ is the ratio of asperity deformation $\omega$ and critical interference $\omega_{c}$. The relationship between the the micro-contact normal load and the contact deformation can be expressed as follows [14]

$$
P=P_{c}\left\{\begin{array}{cc}
\omega^{*} & \omega^{*} \leq 1 \\
1.03 \omega^{* 1.425} & 1<\omega^{*} \leq 6 \\
1.4 \omega^{* 1.263} & 6<\omega^{*} \leq 110
\end{array}\right.
$$

Since the KE model obtained the final equation by using the curve fitting based on the finite element data, the fitting curve on the elastic-plastic boundary is discontinuous.

To solve this problem, Brizmer et al.[15] investigated the effects of the contact conditions (frictionless or fully-adhered) and material properties on the normal contact (the material Poisson's ratio under $0-0.25$ and $0.2-0.5$ conditions), the relationship of contact parameters and normal contact load, (Poisson's ratio under and 0.2-0.5 condition), can be expressed as

$$
P=P_{c} \frac{\overline{l_{c}}}{{\overline{\delta_{c}}}_{c}^{3 / 2}} \begin{cases}\omega^{* 3 / 2} & \omega^{*} \leq \overline{\delta_{c}} \\ \omega^{* 3 / 2} \times\left(1-e^{\bar{\delta}_{c}^{\alpha} /\left({\overline{\delta_{c}}}^{\alpha}-\omega^{* \alpha}\right)}\right) & \omega^{*}>\overline{\delta_{c}}\end{cases}
$$

Where, $\quad \bar{l}_{c}=8.88 v-10.13\left(v^{2}+0.089\right) \quad, \quad \gamma=0.25+0.125 v \quad, \quad \overline{\delta_{c}}=6.82 v-7.83\left(v^{2}+0.0586\right)$, $\alpha=0.174+0.08 v$.

\section{The normal contact damping predict model for joint surface}

When the joint surfaces contact each other under the normal load, the asperity on the rough surface occurs deformation. If the asperity contact area more than the critical contact area, the asperity store elastic strain energy, which show the stiffness characteristic. The asperity occurs plastic deformation and dissipation energy when its contact area does not exceed the critical contact area, and show the damping characteristic.

Substituting Eq. (4) into (6) yields

$\omega_{c}=\left(\frac{K H}{2 E^{*}}\right)^{2} \frac{a^{D / 2}}{G^{D-1}}$

The critical truncated area of asperity can be expressed as (when $\omega=\omega_{c}$ )

$a_{c}^{\prime}=G^{2}\left(\frac{K H}{2 E^{*}}\right)^{2 /(1-D)}$

According to Eqs. (3) and (11), the ratio of asperity deformation $\omega$ and critical interference $\omega_{c}$ can be obtained as

$$
\omega^{*}=\frac{\omega}{\omega_{c}}=\left(\frac{a_{c}^{\prime}}{a^{\prime}}\right)^{(D-1)}
$$

The relationship of normal contact force $P_{e}$ and contact parameters under elastic stage can be yield according to Eqs. (10, 8, 4 and 13) 
$P_{e}=\frac{4 E^{*} G^{D-1}}{3 \pi} \frac{\overline{l_{c}}}{{\overline{\delta_{c}}}^{3 / 2}} a^{\prime(3-D) / 2}$

The normal contact stiffness $k_{n}$ for each elastic micro-contact can be written as

$k_{n}=\frac{d P_{e}}{d \omega}=\frac{d P_{e}}{d a^{\prime}} \cdot \frac{d a^{\prime}}{d \omega}=\frac{4 E^{*}(3-D)}{3 \pi(2-D)} \frac{\bar{l}_{c}}{\bar{\delta}_{c}^{3 / 2}} a^{\prime 1 / 2}$

The whole normal stiffness $K_{N}$ of contact surface can be given as

$$
\begin{aligned}
& K_{N}=\int_{a_{c}}^{a_{l}^{\prime}} k_{n} n\left(a^{\prime}\right) d a^{\prime} \\
& =\frac{4 D E^{*}(3-D)}{3 \pi(2-D)(1-D)} \frac{\overline{l_{c}}}{\bar{\delta}_{c}^{3 / 2}} \psi^{(2-D) / 2} a_{l}^{a^{D / 2}}\left(a_{l}^{(1-D) / 2}-a_{c}^{(1-D) / 2}\right)
\end{aligned}
$$

According to Ref.[11], the ratio of real contact area $A r$ and nominal contact area $A a$ can be expressed as $\quad A_{r}^{*}=\frac{A r}{A a}=\frac{D \psi^{(2-D) / 2} a_{l}^{\prime}}{(2-D) A a}$

Substituting Eq. (17) into (16) yields

$$
K_{N}=\frac{4 D E^{*}(3-D)}{3 \pi(2-D)(1-D)} \frac{\bar{l}_{c}}{\bar{\delta}_{c}^{3 / 2}} \psi^{(2-D) / 2}\left(\frac{(2-D) A a}{D \cdot \psi^{(2-D) / 2}} A r^{*}\right)^{D / 2}\left(\left(\frac{(2-D) A a}{D \cdot \psi^{(2-D) / 2}} A r^{*}\right)^{(1-D) / 2}-a_{c}^{\prime(1-D) / 2}\right)
$$

It occurs elastic deformation for micro-contact when $a^{\prime}>a_{c}^{\prime}$, the elastic energy of a single asperity can be expressed as

$$
w_{n e}=\int_{0}^{\omega} P_{e} d \omega=\frac{4 E^{*} G^{2(D-1)}}{3 \pi} \frac{\overline{l_{c}}}{{\overline{\delta_{c}}}^{3 / 2}} a^{(5-2 D) / 2}
$$

The elastic energy of contact surface can be obtained by integrating Eq. (19) as follow

$$
W_{N E}=\int_{a_{c}^{\prime}}^{a_{l}^{\prime}} w_{n e} n\left(a^{\prime}\right) d a^{\prime}=\frac{4 E^{*} D G^{2(D-1)}}{3 \pi(5-3 D)} \frac{\overline{l_{c}}}{\bar{\delta}_{c}^{3 / 2}} \psi^{(2-D) / 2} a_{l}^{\prime D / 2}\left(a_{l}^{(5-3 D) / 2}-a_{c}^{\prime(5-3 D) / 2}\right)
$$

When $a^{\prime} \leq a_{c}^{\prime}$, the asperity occurs plastic deformation. The plastic energy of one micro-contact under plastic force $P_{p}$ is given by [16]

$$
w_{n p}=\int_{0}^{\omega} P_{p} d x=H a^{\prime} \omega
$$

The whole dissipation of energy in plastic contact region can be obtained as

$$
W_{N P}=\int_{0}^{a_{c}^{\prime}} w_{n p} n\left(a^{\prime}\right) d a^{\prime}=\frac{H D G^{D-1}}{2(2-D)} \psi^{(2-D) / 2} a_{l}^{\prime D / 2} a_{c}^{\prime 2-D}
$$

The normal contact damping dissipation factor can be obtained as

$$
\eta_{n}=\frac{W_{N P}}{W_{N E}}=\frac{\frac{H}{2(2-D)} a_{c}^{\prime 2-D}}{\frac{4 E^{*} G^{(D-1)}}{3 \pi(5-3 D)} \frac{\overline{l_{c}}}{\bar{\delta}_{c}^{3 / 2}}\left(a_{l}^{(5-3 D) / 2}-a_{c}^{\prime(5-3 D) / 2}\right)}
$$

The normal damping of contact surface can be written as $C_{n}=\eta_{n} \sqrt{M K_{n}}$

$$
=\frac{\frac{H}{2(2-D)} a_{c}^{\prime 2-D}}{\frac{4 E^{*} G^{(D-1)}}{3 \pi(5-3 D)} \overline{\bar{l}_{c}}\left({\frac{5}{\delta_{c}}}^{3 / 2}\left(a_{l}^{\frac{5-3 D}{2}}-a_{c}^{\frac{5-3 D}{2}}\right)\right.} \cdot \sqrt{M \frac{4 D E^{*}(3-D)}{3 \pi(2-D)(1-D)} \frac{\overline{l_{c}}}{\bar{\delta}_{c}^{3 / 2}} \psi^{\frac{2-D}{2}} a_{l}^{\frac{D}{2}}\left(a_{l}^{\frac{1-D}{2}}-a_{c}^{\frac{1-D}{2}}\right)}
$$

Where, $M$ is the mass of the structure.

Substituting Eq. (17) into (24) yield 


$$
\begin{aligned}
& C_{n}=\frac{\frac{H}{2(2-D)} a_{c}^{\prime 2-D}}{\frac{4 E^{*} G^{(D-1)}}{3 \pi(5-3 D)} \frac{\bar{l}_{c}}{\bar{\delta}_{c}^{3 / 2}}\left(\left(\frac{(2-D) A a}{\left.\left.\left.D \cdot \psi^{\frac{2-D}{2}} A r^{*}\right)^{\frac{5-3 D}{2}}-a_{c}^{\prime}\right)^{\frac{5-3 D}{2}}\right)}\right.\right.} \\
& \times \sqrt{M \frac{4 D E^{*}(3-D)}{3 \pi(2-D)(1-D)} \frac{\bar{l}_{c}}{\delta_{c}^{3 / 2}} \psi^{\frac{2-D}{2}}\left(\frac{(2-D) A a}{D \cdot \psi^{\frac{2-D}{2}}} A r^{*}\right)^{\frac{D}{2}}\left(\left(\frac{(2-D) A a}{D \cdot \psi^{\frac{2-D}{2}}} A r^{*}\right)^{\frac{1-D}{2}}-a_{c}^{\prime \frac{1-D}{2}}\right)}
\end{aligned}
$$

\section{Numerical Simulation}

As Ref.[17], the fractal dimension and fractal roughness parameter of nodular cast iron (Material type is QT600-3 in China) with roughness $R_{a}=1.6 \mu \mathrm{m}$ can be calculated as $D=1.42, G=1.21 \times 10^{-10}$ respectively. The property parameters of the contact material are $E=150 G P a, H=196{ }^{\circ} H B \quad, v=0.3$, the shear modulus is $107 \mathrm{Gpa}$, unit area $A a=10^{-6} \mathrm{~m}^{2},, M=2.5 \mathrm{Kg}$. Corresponding to the value of $D$, the domain extension factor can be found in Ref.[11],which is $\psi=2.067$.

Substituting parameter values into Eq.(25), the relationship of normal contact damping and the ratio of real contact area and nominal contact area $\left(A_{r}{ }^{*}=A_{r} / A_{a}\right)$ can be shown in the fig.3
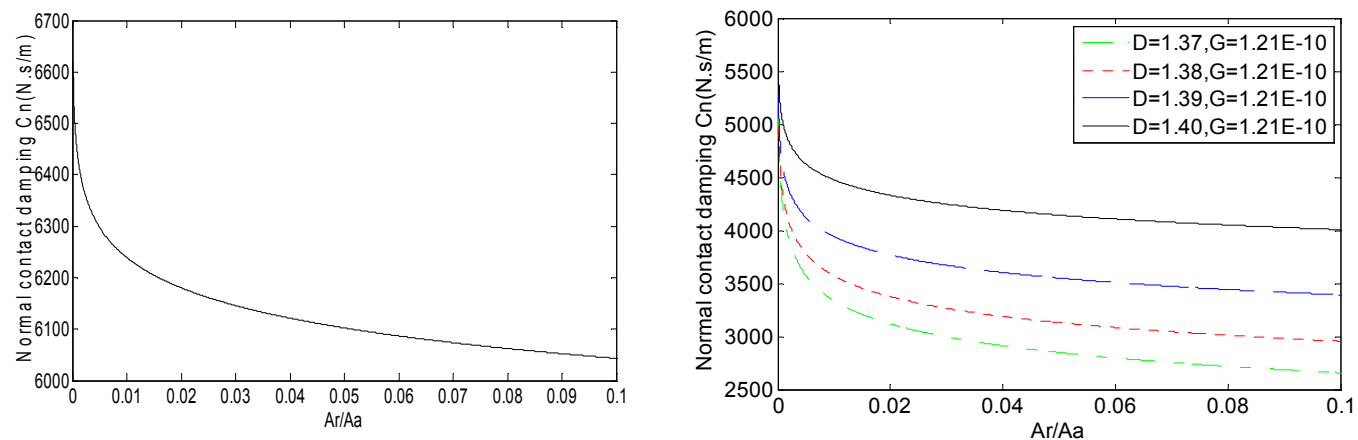

Fig.4 Relationship between normal damping and $A_{r} / A_{a} \quad$ Fig.5 Influence of the fractal dimension

Change the value of the fractal dimension $D$, the effect on the normal contact damping as shown in fig. 5

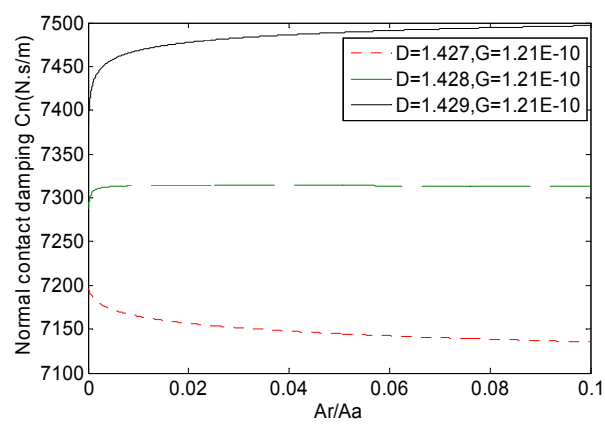

Fig.6 inflection point appear at $D=1.428$

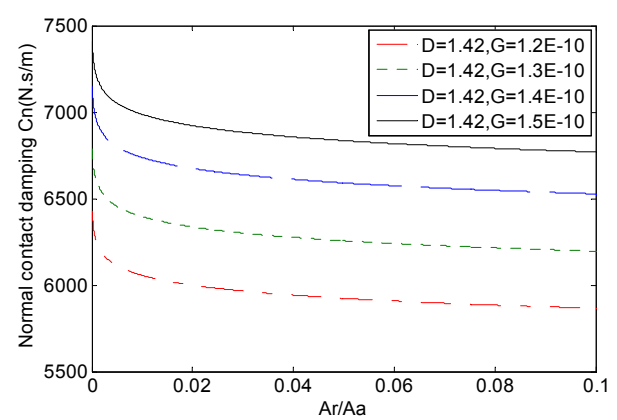

Fig.7 Influence of fractal roughness parameter

With the increasing of fractal dimension, there is a inflection point appear at $D=1.428$ in the simulation. When $D>1.428$, the relationship between normal contact damping and area ratio $\left(A_{r} / A_{a}\right)$ is convex arc nonlinear, and can be shown in Fig.6. The effect of the fractal roughness parameter also can be shown in fig. 7 . 


\section{Conclusion}

When $1<D<1.428$, the normal contact damping and contact area ratio show a concave arc nonlinear relationship, and the normal contact damping decrease with the increase of contact area, increase with the increase of fractal dimension. When $1.428<D<2$, the normal contact damping and contact area ratio show a convex arc nonlinear relationship, and the normal contact damping increase with the increase of contact area, increase with the increase of fractal dimension.

Fractal roughness parameter $\mathrm{G}$ is nonlinear relationship with the normal contact damping, and when the fractal roughness parameter increase, the greater the joint surface normal contact damping is.

The emergence of inflection point provide a reference for the calculation of damping and the optimization design of the contact parameters.

\section{References}

[1] Xu C. Study on nonlinear dynamic response of bolted jointed beam [J]. Structure \& Environment Engineering, Vol. 38(2011) No. 2, p.1-5.

[2] Ibrahim R A, Pettit C L. Uncertainties and dynamic problems of bolted joints and other fasteners [J]. Journal of sound and Vibration, Vol. 279 (2005) No. 3, p. 857-936.

[3] Fu W P, Huang Y M, Zhang X L, et al. Experimental Investigation of Dynamic Normal Characteristics of Machined Joint Surfaces[J]. Journal of Vibration \& Acoustics, Vol. 122 (2000) No. 4, p.393-398.

[4] Ahmadian H, Jalali H. Identification of bolted lap joints parameters in assembled structures [J]. Mechanical Systems \& Signal Processing, Vol. 21 (2007) No. 2, p.1041-1050.

[5] Majumdar A, Bhushan B. Fractal model of elastic-plastic contact between rough surfaces [J]. Journal of Tribology, Vol. 113 (1991) No. 1, p.1-11.

[6] Zhang X, Wang N, Lan G, et al. Tangential Damping and its Dissipation Factor Models of Joint Interfaces Based on Fractal Theory With Simulations[J]. Journal of Tribology, Vol. 136 (2013) No. 1, p.249-256.

[7] You J M, Chen T N. Estimation for Normal Parameters of Joint Surfaces Based on Fractal Theory [J]. Journal of Shanghai Jiaotong University, Vol. 45 (2011) No. 9, p.275-1280.

[8] Zhang X. Normal Contact Damping and Dissipation Factor Model of Joint Interfaces Based on Fractal Theory $[\mathrm{J}]$. Transactions of the Chinese Society for Agricultural Machinery, Vol. 44 (2013) No. 6, p.287-294.

[9] Wan F, Li G X, Gong J Z, et al. An improved algorithm for the normal contact stiffness and damping of a mechanical joint surface $[\mathrm{J}]$. Proceedings of the Institution of Mechanical Engineers Part B Journal of Engineering Manufacture, Vol. 228 (2014) No. 5, p.751-765.

[10]Majumdar, A., and Bhushan, B., Fractal Model of Elastic-Plastic Contact Between Rough Surfaces, ASME J. Tribol.,Vol. 113 (1991) No. 1, p.1-11.

[11] Wang S, Komvopoulos K. A fractal theory of the interfacial temperature distribution in the slow sliding regime: Part I-elastic contact and heat transfer analysis [J]. Journal of Tribology, Vol. 116 (1994) No. 4, p.812-822.

[12]Johnson,K.L. Contact Mechanics[J]. Journal of Tribology, Vol. 108 (1986) No. 4, p.464.

[13]CHANG W R, ETSION I, BOGY D B, Static Friction Coefficient Model for Metallic Rough 
Surfaces, Journal of Tribology,Vol. 110 (1988) No. 1, p.57-63.

[14]KOGUT L, ETSION I. A static friction model for elastic-plastic contacting rough surfaces [J]. Journal of Tribology, Vol. 126 (2004) No. 1, p. 34-40.

[15]BRIZMER V, ZAIT Y, KLIGERMAN Y, et al. The effect of contact conditions and material properties on elastic-plastic spherical contact [J]. Journal of Mechanics of Materials and Structures, Vol. 1 (2006) No. 5, p.865-879.

[16]YAN W, KOMVOPOULOS K. CONTACT ANALYSIS OF ELASTIC-PLASTIC FRACTAL SURFACES [J]. Journal of Applied Physics,Vol. 84 (1998) No. 7, p. 3617-3624.

[17]Zhao Y, et al. Surface contact stress-based nonlinear virtual material method for dynamic analysis of bolted joint of machine tool. Precis Eng (2015), http://dx. doi.org/10. 1016/j. precisioneng. 2015.08.002 